\title{
Disbond Detection through Thermal Testing of Materials under Noisy Observations
}

\author{
Fumio Kojima \\ Mechanical Engineering Department, Osaka Institute of Technology \\ 5-16-1 Ohmiya, Asahi-ku, Osaka 535, Japan
}

\begin{abstract}
Abstarct
A computational method for the disbond detection in aircraft lap joints and in the adhesive joints between aircraft skin and reinforcing doublers is considered using thermographical data. An idea related to method of mappings applies to a parameter estimation problem for a 2-D heat diffusion system under noisy observations. A computational algorithm for estimating the length of disbond is proposed based on the domain decomposition method.
\end{abstract}

\section{Introduction}

Recently, due to the increasing average of aircraft, the demand has grown for the assesment of structural integrity of aerospace materials. The infrared imaging technique allows inspections of a large area of the structure in a short time period. Mathematically, to estimate the structural information with the thermal data can be formulated as a geometrical heat inverse problem. The inverse formulation of 2-D thermal tomography has been discussed in [1] [2] using the idea related to the method of mappings and in [3] using the boundary integral equation method. Application of an artificial neural network to thermal tomography has been studied in [4]. Our efforts in this paper are directed to disbond detection of aircraft systems using thermal data. The presence of disbonds in aircraft lap joints and in the adhesive joints between aircraft skin and reinforcing doublers causes severe damage in passenger airplanes. Hence it is highly and urgently required to develop a method for characterizing and detecting the disbond area in airplanes. In this paper, a method for detecting and estimating the size of disbond is proposed using the idea related to the domain decomposition approach.

\section{Thermographic Inspections}

In thermographic inspections, the heat is injected to the front surface with lasers or IR lamps and then the subsequent surface temperatures can be recorded by the infrared images without intimate contact with the structure. Figure 1 illustrates this thermal technique using the infrard imaging. Sample materials with epoxy bonded area considered here are depicted in Fig. 2. Thermal imaging data can be obtained from a viewed side of material surface in injecting the heat by IR lamp to the same side. For convenience of discus-

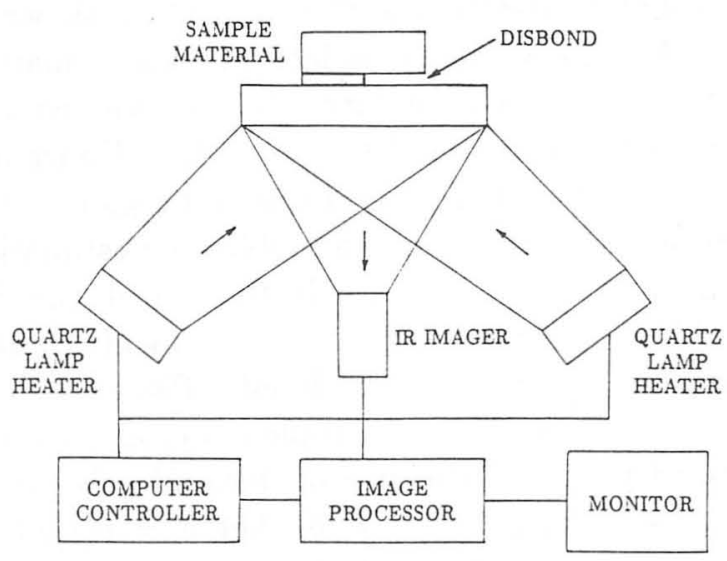

Fig. 1 Infrared measurement system 


\section{Viewed side}

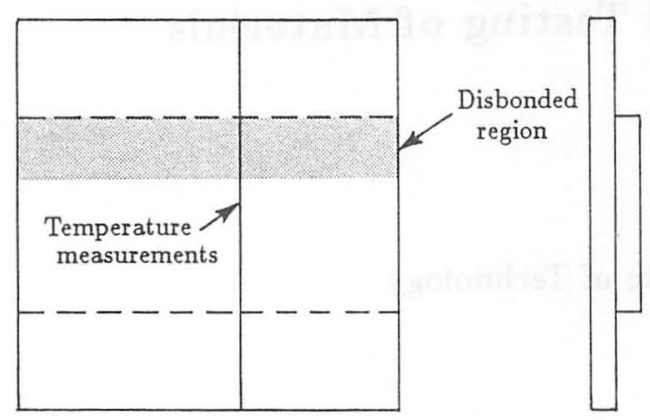

Aluminum with epoxy bondline

Fig. 2 Sample materials

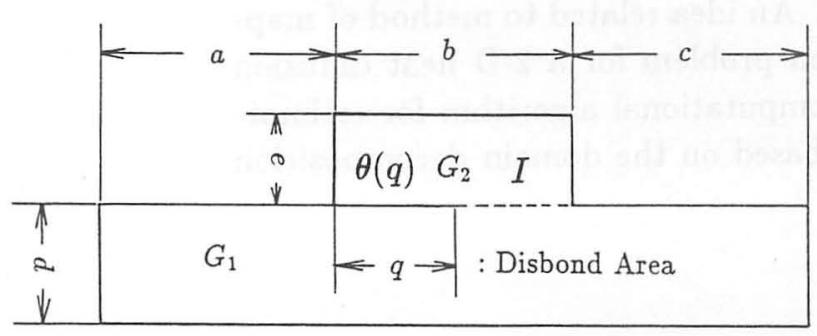

$S$

Fig. 3 Sectional plan of sample materials

sions, we restrict our attention in sectional plan of materials. Suppose that one dimensional thermal data are taken on the line as shown in Fig. 2. Then the input and output relations for this nondestructive measurement system can be described by a two dimensional heat diffusion equation defined on 'T-shaped' domain as shown in Fig. 3. Let $G$ denote the 'T-shaped' domain in two-dimensional Euclidean space. To apply the domain decomposition idea, we decompose $T$-shaped domain into two rectangular subdomains, $G=G_{1} \cup G_{2}$ as shown in Fig. 3. The whole boundary of sample materials is denoted by boundary $\partial G$. The viewed side of materials is denoted by $S(\subset \partial G)$. Under our restriction, the detection of disbond region at the interface is converted into a problem for estimating the length of disbond line. In the sequel, the disbond line at the inteface is denoted by $\theta(q)$ where $q$ implies the length of disbond. Figure 3 illustrates the dimensions of sample materials. For the forward problem, the size of disbond $q$ is preassigned satisfying $0<q<b$. Let $u=u(t, x)$ be the temperature at time $t$ at location $x=\left(x_{1}, x_{2}\right)$. The temperature distribution $u=u(t, \cdot)$ is defined on $G / \theta(q)$ for each time $t$. Then the temperature $u(t, q)$ is described by the solution to the heat diffusion equation,

$$
\frac{\partial u(t)}{\partial t}-\kappa \Delta u(t)=0 \text { in } \mathcal{T} \times G / \theta(q)
$$

with the initial and boundary conditions

$$
\begin{aligned}
& u(0)=u_{0} \text { on } G / \theta(q) \\
& \frac{\partial u(t)}{\partial n}=\left\{\begin{array}{lll}
g / \kappa & \text { on } & S \\
0 & \text { on } & \partial(G / \theta(q)) / S
\end{array} .\right.
\end{aligned}
$$

In the above equations, $\mathcal{T}$ denotes the time interval $\left(0, t_{f}\right)$ during which the process is heated and observed. The boundary input $g$ implies heat source level from the front surface $S$. The coefficient $\kappa$ is the thermal diffusivity of the material inspected. It is noted that the interface condition on the disbond line is replaced by the Neuman boundary condition. Let $\left\{t_{k}\right\}_{k=1}^{n}$ be a discrete time sequence which measurements are made. Then we consider the collection of noisy observations,

$$
Y_{k}=\left.\mathcal{H} u\left(t_{k}, q\right)\right|_{S}+\epsilon_{k} \quad(k=1,2, \cdots, n)
$$

where $Y_{k}$ is an $m$-dimensional data sequence and where the operator $\mathcal{H}$ is associated with the data acquisition by infrared imaging technique. The noise sequence $\left\{\epsilon_{k}\right\}$ is composed of $m$-dimensional independent Gaussian random variables on probability space $(\Omega, \mathcal{F}, P)$, provided with

$$
E\left\{\epsilon_{k}\right\}=0 \quad \operatorname{Var}\left\{\epsilon_{k}\right\}=\sigma^{2} I_{n} .
$$

Hence the joint probability density of $\left\{Y_{k}\right\}_{k=1}^{n}$ is given by

$$
\begin{gathered}
p\left(y_{1}, y_{2}, \cdots, y_{n} ; q\right) \\
=\left(\frac{1}{2 \pi \sigma^{2}}\right)^{\frac{n}{2}} \exp \left[-\sum_{k=1}^{n}\left\{y_{k}-\mathcal{H} u\left(t_{k}, q\right)\right\}^{\prime}\right. \\
\left.\left\{y_{k}-\mathcal{H} u\left(t_{k}, q\right)\right\} / 2 \sigma^{2}\right] .
\end{gathered}
$$

\section{Domain Decomposition Method}

To solve the system (1) numerically, the domain decomposition technique is adopted for saving the computational time. The domain decomposition method is very classical tool for solving elliptic partial differential equations. Recently, there 
has been strong revival of the interest in this approach due to its potential in highly parallel computing environments. There are two alternative methods for this approach (see [6]), i.e., non -overlapping (Schur method) and overlapping method (Schwartz method ). In this paper, due to the geometrical structure of domain, we choose non-overlapping method ( see [5] ). For the convenience of discussions, the finite Galerkin solution can be written by

$$
u^{N}=\left[u_{1}^{N}, u_{2}^{N}, u_{3}^{N}\right]^{T}
$$

where the $u_{i}^{N}$ corresponds to the solution at the node number in $G_{i}(i=1,2)$ and the node number belonging to the interface $I(i=3)$, respectively. The finite Galerkin approximation of (1) can be represented by

$$
C^{N} \frac{d u^{N}(t)}{d t}+A^{N}(q) u^{N}(t)=B^{N}(q) g^{N}(t)
$$

with

$$
u^{N}(0)=\bar{u}_{0}^{N} .
$$

The implicit numerical scheme for the above equation is to solve a linear system of algebraic equation

$$
K^{N}(q) u^{N}(t+\Delta)=b^{N}(t)
$$

at each time step $t=k \Delta\left(k=0,1,2, \cdots, n_{t}\right)$, where

$$
K^{N}(q)=C^{N}(q)+\Delta A^{N}(q)
$$

and

$$
b^{N}(t)=C^{N}(q) u^{N}(t)+\Delta B^{N}(q) g^{N}(t),
$$

respectively. The element matrix can be decomposed into the block matrices,

$$
K^{N}(q)=\left[\begin{array}{ccc}
K_{11}^{N}(q) & 0 & K_{13}^{N}(q) \\
0 & K_{22}^{N}(q) & K_{23}^{N}(q) \\
K_{13}^{N}(q)^{T} & K_{23}^{N}(q)^{T} & K_{33}^{N}(q)
\end{array}\right]
$$

In this decomposition, $K_{i i}^{N}(q)$ represents coupling between the pair of nodes in $\bar{G}_{i}(i=1,2)$ and $I(i=3)$, and $K_{i 3}^{N}(q)(i=1,2)$ imply coupling between the pairs belonging to $\bar{G}_{i}$ and $I$, respectively. Similarly, $b^{N}(t, q)$ is decomposed into three parts:

$$
b^{N}=\left[b_{1}^{N}, b_{2}^{N}, b_{3}^{N}\right]^{T},
$$

corresponding to the node points at $\bar{G}_{1}, \bar{G}_{2}, \bar{I}$, respectively. By applying block-Gaussian elimination to (7), the Schur complement of the element matrix $K^{N}(q)$ is given by

$$
\begin{aligned}
K_{C}^{N}(q) & =K_{33}^{N}(q) \\
& -\sum_{i \leq 2} K_{i 3}^{N}(q)^{T}\left(K_{i i}^{N}(q)\right)^{-1} K_{i 3}^{N}(q) .
\end{aligned}
$$

Using this, we can reduce the system (7) to

$$
K_{C}^{N}(q) u_{3}^{N}=b_{3}^{N}(q)
$$

where

$$
\begin{aligned}
b_{3}^{N}(t, q) & =b_{3}^{N}(t, q) \\
& -\sum_{i \leq 2} K_{i 3}^{N}(q)^{T}\left(K_{i i}^{N}(q)\right)^{-1} b_{i}^{N}(t, q) .
\end{aligned}
$$

Then the solutions of $u_{1}^{N}(t+\Delta)$ and $u_{2}^{N}(t+\Delta)$ can be obtained by solving

$$
\begin{gathered}
K_{i i}^{N}(q) u_{i}^{N}(t+\Delta)=b_{i}^{N}(t, q)-K_{33}^{N}(q) u_{3}^{N}(t+\Delta) \\
\text { for } \quad i=1,2 .
\end{gathered}
$$

The size of the element matrix $K_{C}^{N}$ is relatively small, compared with the number of nodes in $G_{i}$. Hence the computational saving can be achieved using an appropriate linear algebraic solver. To solve (9), we use the iterative method based on the conjugate gradient algorithm. We note that the use of direct method by the Cholesky decomposition in (9) requires heavily computational volumes due to the computation of inverse matrices of diagonal block $K_{i i}$ in (8).

\section{Disbond Detection}

The proposed algorithm consists of two parts. The first part of our algorithm is devoted to determining the region of interest in inspections. Secondly, we propose a method for seeking the exact length of disbond on that region.

\subsection{Region of Interest}

Let $Q$ be the admissible set defined by

$$
\begin{gathered}
Q=\left\{q_{1}, q_{2}, \cdots, q_{L} \mid\right. \\
\left.0<\bar{q}^{1} \leq q_{1} \leq \cdots \leq q_{L} \leq \bar{q}^{2}<b\right\}
\end{gathered}
$$


where $q_{i}$ implies the size of disbond. For each $q=q_{i}(i=1,2, \cdots, L)$, the corresponding numerical solution $u=u^{N}\left(t, q_{i}\right)$ can be solved by the domain decomposition method. Thus we collect the event set $\left\{\mathcal{H}^{N} u^{N}\left(\cdot, q_{i}\right)\right\}_{i=1}^{L}$ and the likelihood test is performed in the following manner:

Given $Q$ and the observed data set $Y_{n}=\left\{y_{k}\right\}_{k=1}^{n}$, choose $\tilde{q} \in Q$ such that

$$
\max _{1 \leq i \leq L} \log p^{N}\left(Y_{n} ; q_{i}\right)
$$

where

$$
\begin{gathered}
p^{N}\left(y_{1}, y_{2}, \cdots, y_{n} ; q\right)=\left(\frac{1}{2 \pi \sigma^{2}}\right)^{\frac{n}{2}} \\
\times \exp \left[-\sum_{k=1}^{n}\left\{y_{k}-\mathcal{H}^{N} u^{N}\left(t_{k}, q\right)\right\}^{\prime}\right. \\
\left.\left\{y_{k}-\mathcal{H}^{N} u^{N}\left(t_{k}, q\right)\right\} / 2 \sigma^{2}\right] .
\end{gathered}
$$

Although the solution $\tilde{q}$ become optimal in the sense of maximum likelihood estimator, this value does not mean the exact length of disbond because of the finite set of admissible class $Q$. More precisely, the value of $\tilde{q}$ provides the reasonable information about the neighborhood of the origin of disbond line. Suppose that the true length of disbond is given by $q_{o}$ and that the optimal solution $\tilde{q}$ is $q_{j}, j \in\{1,2, \cdots L\}$. Then we may argue that

$$
q_{o} \in I \subset\left(q_{j-1}, q_{j+1}\right) .\left(q_{0}=\bar{q}^{1}, q_{L+1}=\bar{q}^{2}\right)
$$

where $I$ is the region of interest in inspections.

\subsection{Parameter Estimation}

In this section, a feasible method is considered for more careful estimation of the size of disbond. Applying the method of mapping technique to 'Tshaped' domain, the size of disbond $q$ can be represented as coefficients in the system equation (1) while the domain $G / \theta(q)$ is replaced by the fixed domain $G / \theta(\tilde{q})$. In the following discussions, we assume that the optimal solution $\tilde{q}$ exists. Namely the unknown parameter $q$ is defined on the interval $I$. Let $\tilde{q}$ be a reference line corresponding to the unknown parameter $q$. We introduce a mapping operator from $\bar{G}_{1}$ into $G_{1}(q)$,

$$
T_{1}(q):\left\{\begin{array}{l}
x_{1}=f_{1}\left(q, z_{1}\right) \\
x_{2}=z_{2}
\end{array}\right.
$$

where

$$
f_{1}\left(q, z_{1}\right)=\left\{\begin{array}{l}
z_{1}\left(0 \leq z_{1} \leq a\right) \\
q\left(z_{1}-a\right) / \tilde{q}+a \\
\left(a<z_{1} \leq a+\tilde{q}\right) \\
(b-q)\left(z_{1}-a-b\right) /(b-\tilde{q})+a \\
+b\left(a+\tilde{q}<z_{1} \leq a+b\right) \\
z_{1}\left(a+b<z_{1} \leq a+b+c\right)
\end{array} .\right.
$$

Similarly, a mapping operator from subdomain $G_{2}$ into $\bar{G}_{2}$ is described as follows:

$$
T_{2}(q):\left\{\begin{array}{l}
x_{1}=f_{2}\left(q, z_{1}\right) \\
x_{2}=z_{2}
\end{array}\right.
$$

where

$$
f_{2}\left(q, z_{1}\right)=\left\{\begin{array}{l}
q z_{1} / \text { tildeq }\left(0<z_{1} \leq \tilde{q}\right) \\
(b-q)\left(z_{1}-b\right) /(b-\tilde{q})+b \\
\left(\tilde{q}<z_{1} \leq b\right)
\end{array} .\right.
$$

Figure 4 illustrates those mappings, $T_{1}(q)$ and $T_{2}(q)$,respectively. Let us define the system state on the reference domain $\bar{G}_{1} \cup \bar{G}_{2}$,

$$
\tilde{u}_{i} \stackrel{\text { def }}{=} u \circ T_{i}(q) \quad(i=1,2) .
$$

Then the transformed system can be described by

$$
\sum_{i \leq 2}\left\{\left\langle\frac{d \tilde{u}_{i}}{d t}, \phi\right\rangle_{L^{2}\left(\bar{G}_{i}\right)}+a_{i}(q)\left(\tilde{u}_{i}(t), \phi\right)\right\}=L(q)(\phi)
$$

$$
\text { for } \forall \phi \in H^{1}\left(\bar{G}_{1} \cup \bar{G}_{2}\right)
$$

with

$$
\tilde{u}_{i}(0)=\bar{u}_{0} \circ T_{i}(q)
$$

where $a_{i}(q)(\cdot, \cdot)$ and $L(q)(\cdot)$ denote, respectively, a sesquilinear form on $H^{1}\left(\bar{G}_{i}\right) \times H^{1}\left(\bar{G}_{i}\right)$ and a linear form on $H^{1}\left(\bar{G}_{1}\right)$ such that, for $\phi, \psi \in H^{1}\left(\bar{G}_{1} \cup \bar{G}_{2}\right)$,

$$
\begin{gathered}
a_{i}(q)(\phi, \psi) \stackrel{\text { def }}{=} \\
c \iint_{\bar{G}_{i}}\left\{\left(\frac{d f_{i}(q)}{d z_{1}}\right)^{-2} \frac{\partial \phi}{\partial z_{1}} \frac{\partial \psi}{\partial z_{1}}+\frac{\partial \phi}{\partial z_{2}} \frac{\partial \psi}{\partial z_{2}}\right\} d z
\end{gathered}
$$

and

$$
L(q)(\phi) \stackrel{\text { def }}{=} \int_{0}^{a+b+c} \tilde{g} \frac{d f_{1}(q)}{d z_{1}} d z_{1} .
$$



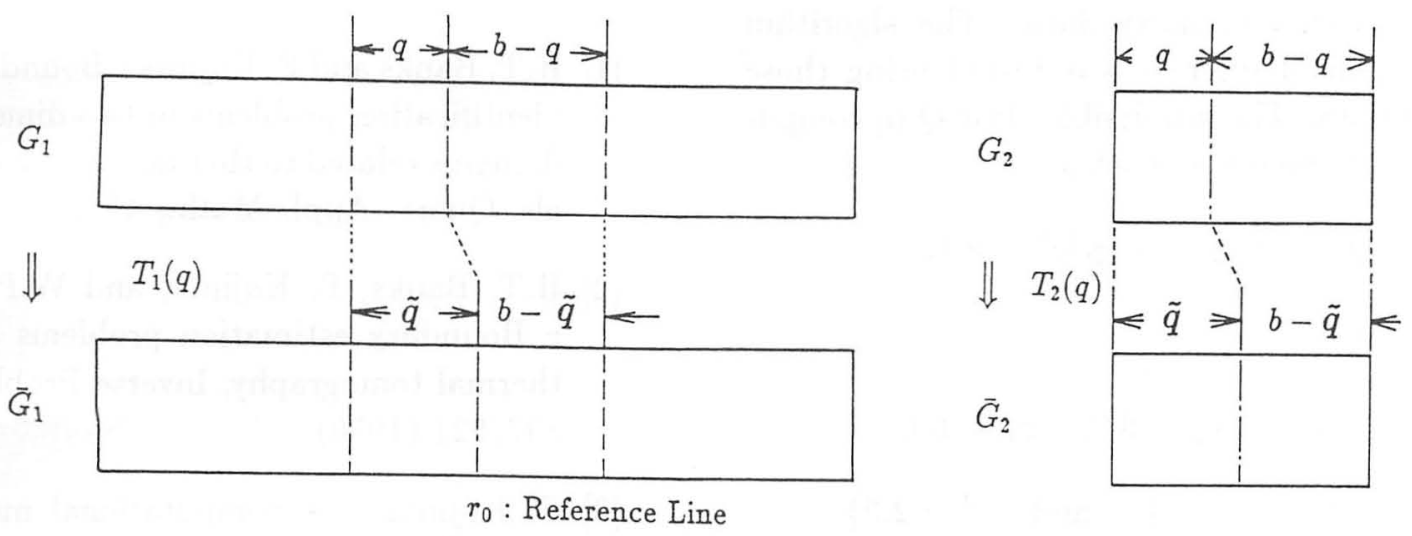

Fig. 4 Method of mappings

The above system can be solved by the same procedures as in the previous section. Thus our parameter estimation problem is to find an optimal solution of

$$
\log \tilde{p}^{N}\left(Y_{n} ; \hat{q}\right)=\max _{q \in I} \log \tilde{p}^{N}\left(Y_{n} ; q\right)
$$

where $\tilde{p}^{N}$ is the likelihood functional corresponding to the numerical solution of the system (13), i. e.,

$$
\begin{gathered}
\tilde{p}^{N}\left(y_{1}, y_{2}, \cdots, y_{n} ; q\right)=\left(\frac{1}{2 \pi \sigma^{2}}\right)^{\frac{n}{2}} \\
\times \exp \left[-\sum_{k=1}^{n}\left\{y_{k}-\mathcal{H}^{N} \tilde{u}_{1}^{N}\left(t_{k}, q\right)\right\}^{\prime}\right. \\
\left.\left\{y_{k}-\mathcal{H}^{N} \tilde{u}_{1}^{N}\left(t_{k}, q\right)\right\} / 2 \sigma^{2}\right] .
\end{gathered}
$$

Since the analytic gradient of cost can be easily evaluated, various optimization routines are available for solving (14). In our algorithm, the quasiNewton method with BFGS-secant approximation for Hessian was used.

\section{Computational Experiments}

In a series of experiments, the algorithm proposed was tested using the simulation data. An alminum sample with epoxy bondline was considered as depicted in Fig. 2. A material sample with dimensions $6.0 \times 0.04$ (inch) (the subdomain $\bar{G}_{1}$ ) and $3.0 \times 0.04$ (inch) (the subdomain $\bar{G}_{2}$ ) was used, i. e.,

$$
\begin{gathered}
a=1.5, \quad b=3.0, \quad c=1.5, \\
\text { and } \quad d=e=0.04
\end{gathered}
$$

The thermal diffusivity coefficient in these experiments was $\kappa=0.12197\left((\text { inch })^{2} / s e c\right.$ ). To discretize the system model by a bilinear finiteelement method, each subdomain $\bar{G}_{j}(j=1,2)$ is divided into a finite number of elements $\left\{e_{k}\right\}_{j=1}^{K_{j}}$ and a number $N_{j}\left(\geq K_{j}\right)$ of nodes defined by $\left\{z_{i}\right\}_{i=1}^{N_{j}}$ are selected in $\bar{G}_{j}$. Each element is preassigned as an axiparallel rectangle with nodes at the vertices. The number of finite elements and nodes in computational experiments were set as

$$
\begin{array}{ll}
K_{1}=32 \times 2 & N_{1}=33 \times 3 \\
K_{2}=16 \times 2 & N_{2}=17 \times 3
\end{array}
$$

Integration in the element matrices and vectors can be computed through piecewise bilinear basis functions. The initial state and the boundary input were preassigned as $u_{0}=0$. and $g=1.0$, respectively. The discrete state model can be solved with respect to discrete time $t=k \Delta$ $(k=0,1, \cdots, n)$ where $\Delta=t_{f} / n$. The initial and final time, and number of time divisions were taken as $t_{f}=19.2(\mathrm{sec})$ and $n=64$ in our computations. The model output data are discretized into thirty spatial points at each sampling time. For these test computations, simulated data $Y_{n}$ were generated by first solving the finite-element model with the same number of finite elements and nodes. Random Gaussian noise at various levels 
was then added to the numerical solution, thereby producing simulated noisy data. The algorithm for the region of interest was tested using those simulated data. The admissible class $Q$ in computational experiments was set as

$$
Q=\left\{q_{1}, q_{2}, \cdots, q_{5} \mid \bar{q}^{1}=0.1,\right.
$$

where

$$
\begin{gathered}
q_{1}=0.4, \quad q_{2}=0.9, \quad q_{3}=1.4 \\
\left.q_{4}=1.9, \quad q_{5}=2.4, \quad \text { and } \quad \bar{q}^{2}=2.9\right\}
\end{gathered}
$$

respectively. The event set

$$
\left\{\mathcal{H}^{N} u^{N}\left(\cdot, q_{i}\right)\right\}_{i=1}^{5}
$$

were provided solving the corresponding finite element model. The simulated data were generated with respect to the true parameters as shown in Table 1. For each $q_{o}$, the optimal parameter $\tilde{q}$ is also shown in the same table. Figure 5 reports the correction rate at various noise levels with respect to a hundred trials.

Table 1 Experimental Conditions and Results

\begin{tabular}{|l|l|l|}
\hline & $(1)$ & $(2)$ \\
\hline True value $q_{o}$ & 1.1 & 2.2 \\
Optimal value $\tilde{q}$ & 0.9 & 2.4 \\
Region of Interest $I$ & $(0.4,1.4)$ & $(1.9,2.9)$ \\
\hline
\end{tabular}

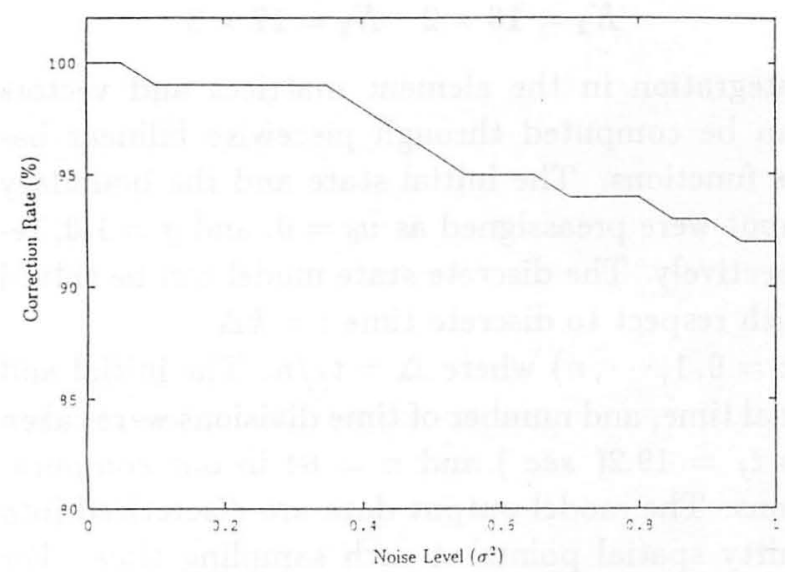

(1)

\section{References}

[1] H.T. Banks and F. Kojima: Boundary shape identification problems in two-dimensional domains related to thermal testing of materials, Quart. Appl. Math., 47 273/293 (1989)

[2] H.T. Banks, F. Kojima, and W.P. Winfree : Boundary estimation problems arising in thermal tomography, Inverse Problems, 6 $897 / 921$ (1990)

[3] F. Kojima : A computational method for thermal tomography by time dependent boundary integral equation method, International Series of Numerical Mathematics, Birkhäuser, 100 207/217 (1991)

[4] F. Kojima : Backpropagation learning using the trust region algorithm and application to nondestructive testing in applied electromagnetics, International Journal of Applied Electromagnetics in Materials, 4 27/33 (1993)

[5] P.E. Bjorstad and B.O. Wildlund : Iterative methods for the solution of elliptic problems on regions partitioned into substructures, SIAM J. Numer. Anal., 23 1097/1120 (1986)

[6] T.F. Chan and D. Goovaerts : Schwartz = Schur : Overlapping versus nonoverlapping domain decomposition, Submitted to SIAM J. Math. Anal.

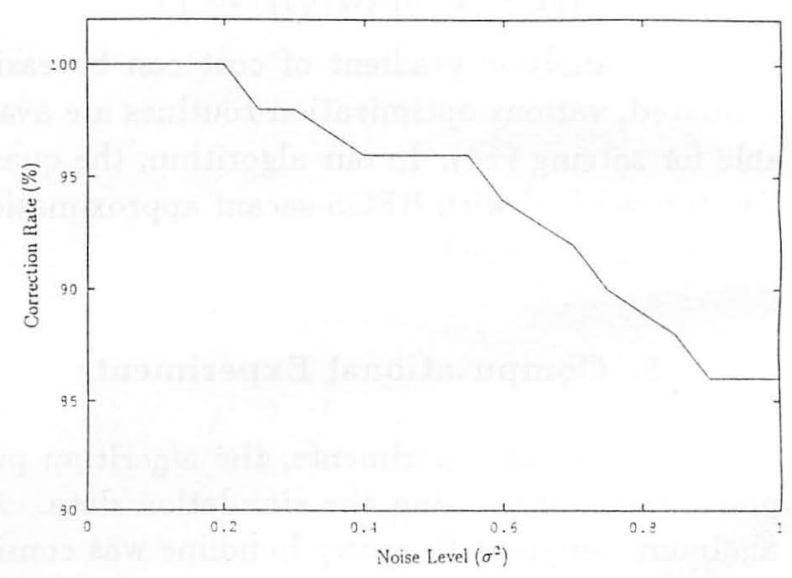

(2)

Fig. 5 Correction rate vs noise level 